\title{
Efficacy of ribavirin against malignant glioma cell lines
}

\author{
AKIYOSHI OGINO $^{1}$, EMIKO SANO ${ }^{2}$, YUSHI OCHIAI ${ }^{1}$, SHUN YAMAMURO $^{1}$, SHINYA TASHIRO ${ }^{2,3}$, \\ KAZUNARI YACHI ${ }^{1}$, TAKASHI OHTA ${ }^{1}$, TAKAO FUKUSHIMA ${ }^{1}$, YUTAKA OKAMOTO $^{4}$, \\ KOUHEI TSUMOTO $^{2,3}$, TAKUYA UEDA ${ }^{2}$, ATSUO YOSHINO ${ }^{1}$ and YOICHI KATAYAMA ${ }^{1}$ \\ ${ }^{1}$ Department of Neurological Surgery, Nihon University School of Medicine, Tokyo 173-8610; ${ }^{2}$ Department of \\ Medical Genome Sciences, Graduate School of Frontier Sciences, The University of Tokyo, Chiba 277-8562; \\ ${ }^{3}$ Medical Proteomics Laboratory, The Institute of Medical Science, The University of Tokyo, Tokyo 108-8639; \\ ${ }^{4}$ New Energy and Industrial Technology Development Organization, Kawasaki 212-8554, Japan
}

Received April 23, 2014; Accepted August 15, 2014

DOI: $10.3892 / 01.2014 .2569$

\begin{abstract}
Ribavirin (1- $\beta$-D-ribofuranosy-1,2,4-triazole3 -carboxamide) has been widely administered as an antiviral agent against RNA and DNA viruses. Ribavirin, in combination with interferon, has predominantly been applied in the treatment of the hepatitis $\mathrm{C}$ virus infection and its potential antitumor efficacy has recently become a point of interest. The aim of the present study was to evaluate the effect of ribavirin on the growth of malignant glioma cells, to identify novel predictive genes in malignant glioma cells (by analyzing gene expression profiles) and to assess the influence of ribavirin on the cell cycle of malignant glioma cells. The present study evaluated the antitumor efficacy of ribavirin against various malignant glioma cell lines (A-172, AM-38, T98G, U-87MG, U-138MG, U-251MG and YH-13). After culturing the cells in ribavirin-containing culture medium (final concentration, $0-1,000 \mu \mathrm{M}$ ) for $72 \mathrm{~h}$, the viable proliferated cells were harvested and counted. The half maximal inhibitory concentration of ribavirin, with regard to the growth of the malignant glioma cell lines, was determined from the concentration of ribavirin required for 50\% growth inhibition in comparison to the untreated control cells. Furthermore, the current study identified the genes in which the gene expression levels correlated with the ribavirin sensitivity of the malignant glioma cells lines, using a high-density oligonucleotide array. Finally, cell cycle analysis was performed on the U-87MG cell line. It was identified that ribavirin inhibited the growth of all of the malignant glioma cell lines in a dose-dependent manner, although the ribavirin sensitivity varied between each cell line. Of the extracted genes, PDGFRA demonstrated the strongest positive correlation between gene expression level and ribavirin
\end{abstract}

Correspondence to: Dr Takao Fukushima, Department of Neurological Surgery, Nihon University School of Medicine, 30-1 Oyaguchi-Kamimachi, Itabashi-ku, Tokyo 173-8610, Japan E-mail: fukushima27takao@gmail.com

Key words: ribavirin, malignant glioma sensitivity. Cell cycle analysis of the U-87MG cell line demonstrated that ribavirin treatment induces G0/G1 arrest and thus may be an effective agent for inhibiting malignant glioma cell growth. Therefore, the results of the current study indicate that ribavirin may have potential as a therapeutic agent in the treatment of malignant gliomas.

\section{Introduction}

Ribavirin (1- $\beta$-D-ribofuranosy-1,2,4-triazole-3-carboxa mide) was developed as an antiviral agent against RNA and DNA viruses and its function was first described in 1972 by Sidwell et al (1). Ribavirin, in combination with interferon (IFN), is one of the standard treatment strategies for hepatitis $\mathrm{C}$ virus (HCV) infections (2). Ribavirin was expected to be clinically advantageous in the treatment of various viral infections, however, to date the clinical utilization of ribavirin has been limited to the treatment of HCV infections.

Recently, interest in the efficacy of ribavirin for the treatment of tumors has increased due to two reasons. Firstly, ribavirin inhibits inosine-5'-monophosphate dehydrogenase (IMPDH) (3). IMPDH is a key enzyme in guanosine triphosphate synthesis, and IMPDH expression levels and activity are elevated in certain types of tumor. Furthermore, IMPDH is also associated with the proliferation and transformation of malignant tumor cells (4). Therefore, an IMPDH inhibitor may be a candidate for antitumor chemotherapy. Secondly, ribavirin inhibits the eukaryotic translation initiation factor 4E (eIF4E) $(5,6)$. eIF4E has two major functions in gene expression: Messenger (m)RNA translation and mRNA export (7). The eIF4E protein is present in the nucleus and the cytoplasm; in the nucleus, eIF4E facilitates the export of a subset of specific growth-promoting mRNAs. In the cytoplasm, eIF4E recruits mRNA with highly structured 5 -untranslated regions to the ribosome to promote translation. Therefore, eIF4E, which is overexpressed in $\sim 30 \%$ of human cancers $(5,8)$, may have oncogenic potential.

The antitumor efficacy of ribavirin has been reported in breast cancer and leukemia $(5,9)$, however, to the best of our knowledge it has yet to be reported in malignant glioma. The aim of the present investigation was to evaluate the antitumor 
efficacy of ribavirin on malignant glioma cells, to identify novel predictive genes for ribavirin sensitivity through the evaluation of gene expression profiles and to assess the influence of ribavirin on the cell cycle of malignant glioma cells.

\section{Materials and methods}

Cell lines and cell culture. Human malignant glioma cells of the A-172, AM-38, T98G, U-251MG and YH-13 cell lines were obtained from Health Science Research Resources Bank (Osaka, Japan), and the U-87MG and U-138MG cell lines were purchased from the American Type Culture Collection (Manassas, VA, USA). All of the cell lines were cultured in Dulbecco's modified Eagle's medium (Nissui Pharmaceutical, Tokyo, Japan) supplemented with $10 \%$ fetal bovine serum (Life Technologies, Grand Island, NY, USA) in a standard humidified incubator at $37^{\circ} \mathrm{C}$ with an atmosphere of $5 \% \mathrm{CO}_{2}$.

Cell culture growth with ribavirin. Malignant glioma cell proliferation was evaluated using a Z1 Coulter Counter ${ }^{\circledR}$ (Beckman Coulter, Brea, CA, USA) to count the cell growth in 24-well plates (Iwaki, Chiba, Japan) . Each well was seeded with $1 \times 10^{4}$ cells and cultured for $24 \mathrm{~h}$ prior to ribavirin treatment to allow adherence of the cells to the plate. The culture medium was replenished with fresh medium containing ribavirin $(0.1-1,000 \mu \mathrm{M})$, and the cells were cultured for $72 \mathrm{~h}$. The proliferated cells were trypsinized with trypsin-EDTA solution (Invitrogen Life Technologies, San Diego, CA, USA) and counted using the $\mathrm{Z} 1$ Coulter Counter ${ }^{\circledR}$. The cell culture growth experiments were repeated a minimum of seven times at each concentration. The half maximal inhibitory concentration $\left(\mathrm{IC}_{50}\right)$, with regard to the growth of the malignant glioma cell culture, was determined from the concentration of ribavirin required for $50 \%$ growth inhibition in comparison to untreated control cells.

RNA preparation and hybridization. Total RNA was extracted from the malignant glioma cells using an RNeasy ${ }^{\circledR}$ mini kit (Qiagen, Valencia, CA, USA) and quantified by spectrophotometry (Jasco V-550 spectrophotometer; Jasco Interntational Co., Ltd., Tokyo, Japan). Double-stranded complementary (c)DNA was generated from the total RNA $(5 \mu \mathrm{g})$ using a One-Cycle cDNA Synthesis kit (Affymetrix, Inc., Santa Clara, CA, USA). Biotinylated cRNA was then synthesized from the cDNA in an in vitro transcription reaction (IVT) by employing an IVT labeling kit (Affymetrix, Inc.). The biotinylated cRNA $(10 \mu \mathrm{g})$ was fragmented and hybridized to a DNA oligonucleotide expression array (Human Genome U133A 2.0 Array; Affymetrix, Inc.) containing $>22,277$ probe sets for $\sim 14,500$ human genes (certain genes are represented on the array by multiple probe sets). The hybridized probe array was washed and stained with a streptavidin-phycoerythrin conjugate (Molecular Probes Life Technologies, Carlsbad, CA, USA) using a GeneChip ${ }^{\circledR}$ Fluidics Station 450 (Affymetrix, Inc.) according to the manufacturer's instructions, as previously described $(10,11)$.

Identification of discriminatory genes for ribavirin sensitivity. The probe array was scanned with a confocal laser scanner

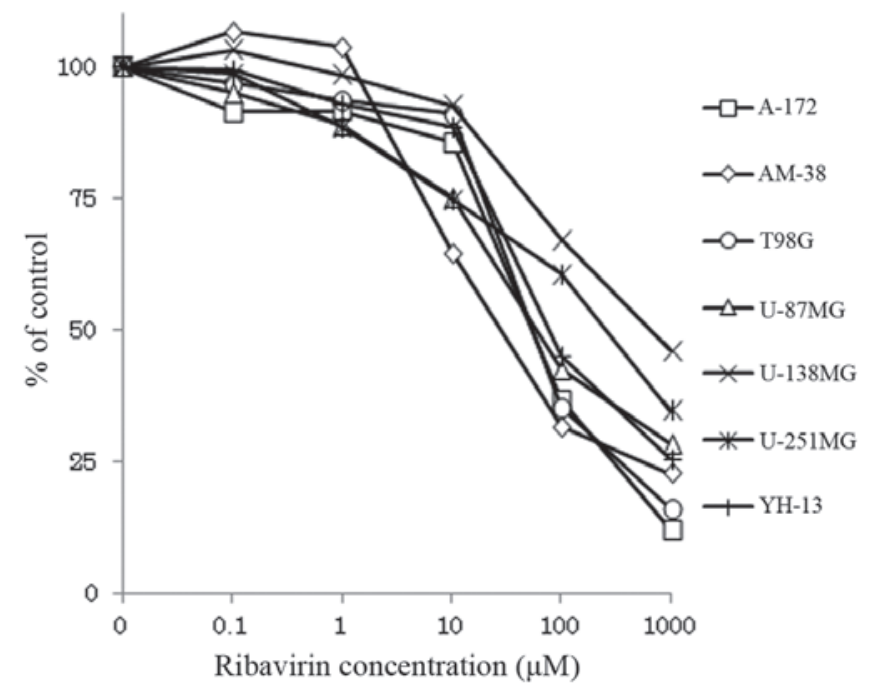

Figure 1. Antitumor effect of ribavirin on seven malignant glioma cell lines. At $72 \mathrm{~h}$, following the addition of ribavirin (final concentration, $0-1,000 \mu \mathrm{M}$ ) to the culture medium, the number of viable cells was counted and expressed as a percentage of the untreated control cells.
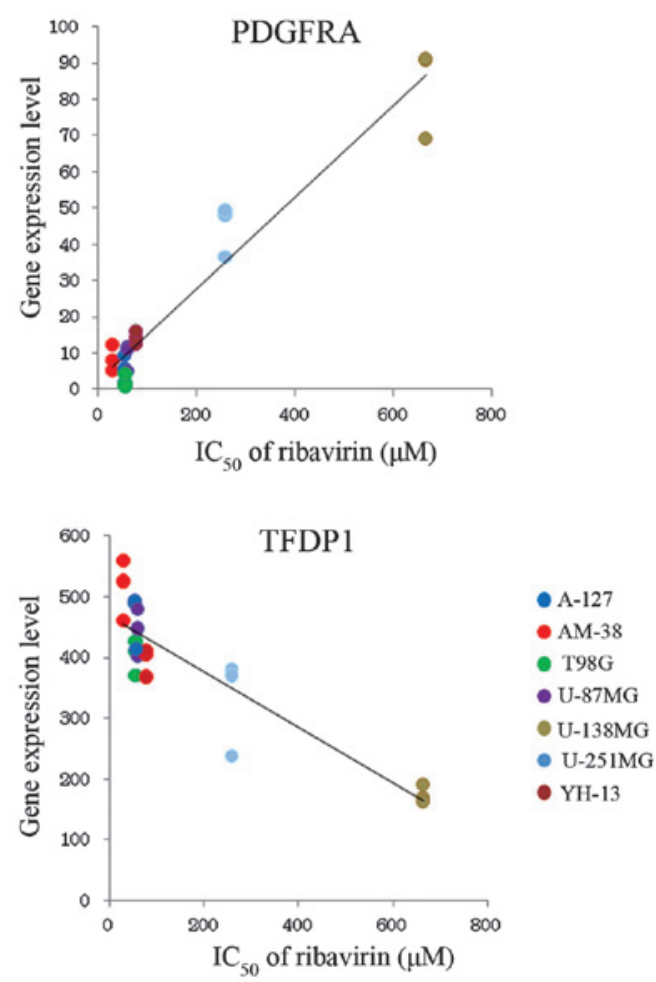

Figure 2. Association between gene expression levels (PDGFRA and TFDP1) and ribavirin sensitivity $\left(\mathrm{IC}_{50}\right.$ of ribavirin) of seven malignant glioma cell lines. $\mathrm{IC}_{50}$, half maximal inhibitory concentration.

(GeneChip ${ }^{\circledR}$ Scanner 3000; Affymetrix, Inc.) and analyzed using GeneChip ${ }^{\circledR}$ Operating Software 1.1 (Affymetrix, Inc.), allowing the gene expression levels to be calculated from the signal intensities. All of the genes represented on the GeneChip ${ }^{\circledR}$ were globally normalized and scaled to the signal intensity to provide a gene expression value. To avoid 
A
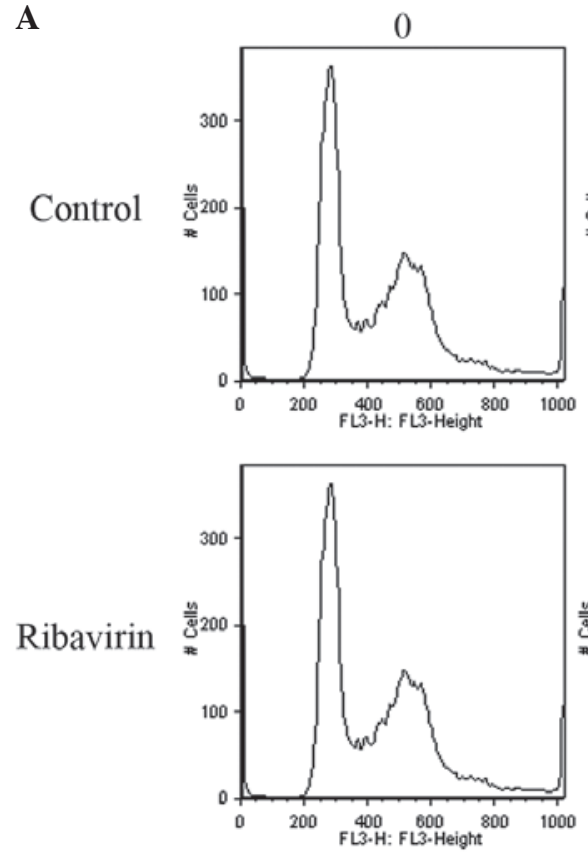

B

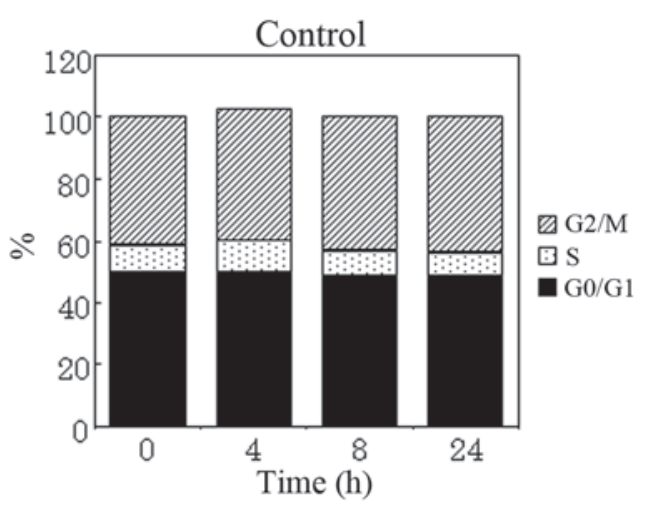

8
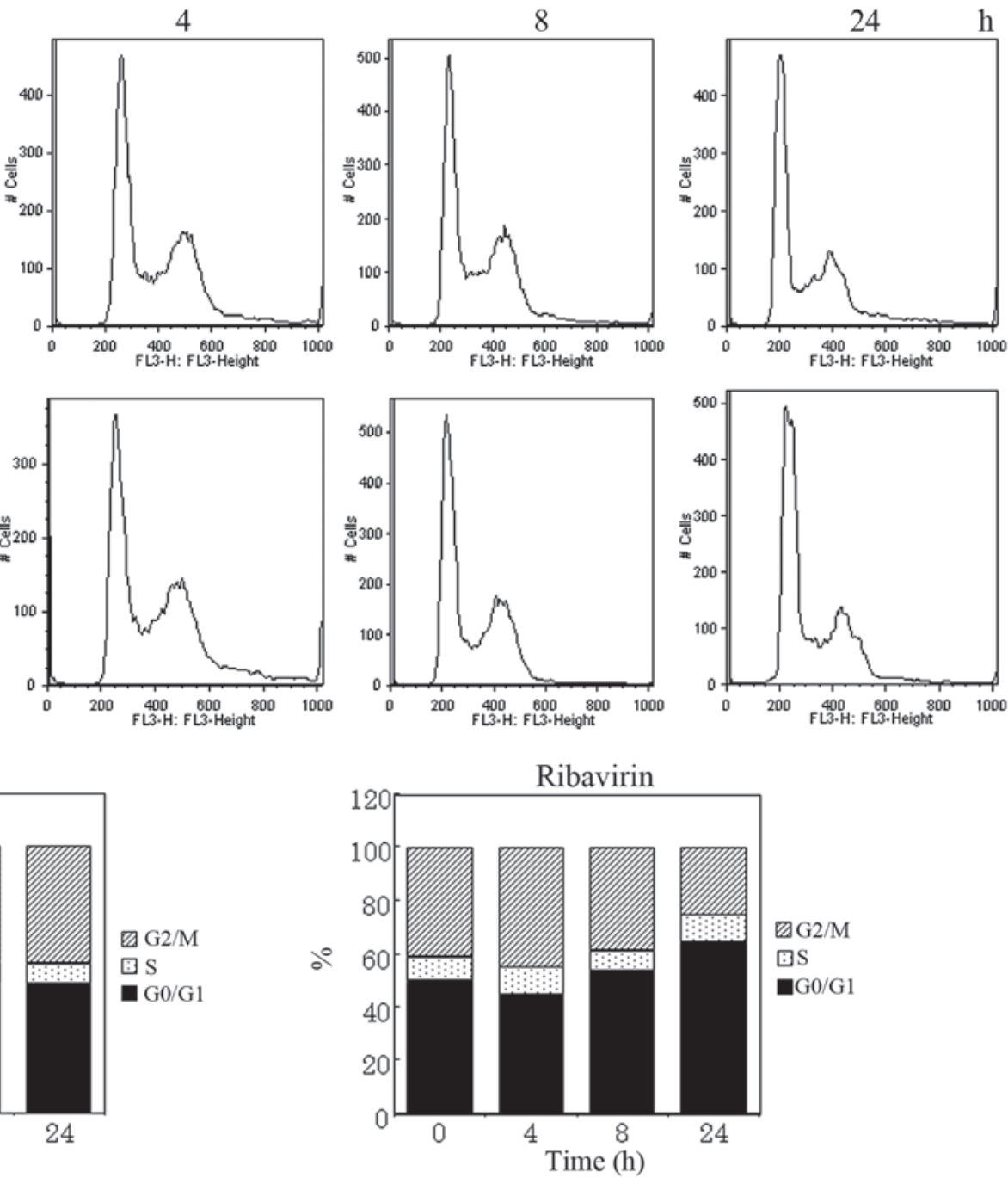

Figure 3.Cell cycle distribution analysis of U-87MG cells, which were treated with ribavirin (with untreated cells serving as the control). (A) Fluorescence-activated cell sorting plot and (B) histograms indicating the antitumor effects.

contributions from artificial sources of variation in the experimentally measured expression patterns, each cell line was grown in three independent cultures and the entire process was conducted independently on mRNA that was extracted from each culture. The expression array analysis for each cell line was then run in triplicate.

Cell cycle distribution analysis. The cells were plated at $2 \times 10^{5}$ cells per $25-\mathrm{cm}^{2}$ flask (Iwaki) and incubated for $24 \mathrm{~h}$ to allow attachment of the cells to the flask. The culture medium was then replenished with fresh medium containing $10 \mu \mathrm{M}$ ribavirin and the cells were harvested using trypsin-EDTA solution at $0,4,8$ and $24 \mathrm{~h}$. The cells were rinsed with phosphate-buffered saline, fixed using ice-cold $70 \%$ ethanol, incubated with $200 \mu \mathrm{g} / \mathrm{ml}$ RNase A (Roche Diagnostics, Basel, Switzerland) for $30 \mathrm{~min}$ at room temperature and stained with $2 \mu \mathrm{g} / \mathrm{ml}$ propidium iodide (PI) solution (Miltenyi Biotec, Inc., San Diego, CA, USA). The fluorescence was measured by flow cytometry, using the BD FACSCalibur ${ }^{\mathrm{TM}}$ flow cytometer (BD Biosciences, Franklin Lakes, NJ, USA), at a fluorescence wavelength of $610 \mathrm{~nm}$. The resulting DNA histogram was analyzed using FlowJo software (BioLegend, Inc., San Diego, CA, USA).
Table I. $\mathrm{IC}_{50}$ of ribavirin for malignant glioma cell lines.

\begin{tabular}{lc}
\hline Cell line & $\mathrm{IC}_{50}, \mu \mathrm{M}$ \\
\hline A-172 & 53.6 \\
AM-38 & 27.9 \\
T98G & 55.0 \\
U-87MG & 59.7 \\
U-138MG & 664.2 \\
U-251MG & 257.7 \\
YH-13 & 76.9 \\
\hline
\end{tabular}

$\mathrm{IC}_{50}$, half maximal inhibitory concentration.

Statistical analysis. To identify the discriminative genes for ribavirin sensitivity, the Pearson's correlation test was performed to evaluate the association between the $\mathrm{IC}_{50}$ of ribavirin and the gene expression level of each gene (Microsoft Office Excel 2007, Redmond, WA, USA). 
Table II. Differentially expressed genes associated with ribavirin sensitivity.

A, Positive differentially expressed genes

\begin{tabular}{|c|c|c|c|}
\hline Symbol & Gene title & Correlation & P-value \\
\hline PDGFRA & Platelet-derived growth factor receptor, $\alpha$ polypeptide & 0.968 & $<0.0001$ \\
\hline ZFP36L2 & Zinc finger protein $36, \mathrm{C} 3 \mathrm{H}$ type-like 2 & 0.951 & $<0.0001$ \\
\hline UBL3 & Ubiquitin-like 3 & 0.944 & $<0.0001$ \\
\hline$H S P B 2$ & Heat shock $27 \mathrm{kDa}$ protein 2 & 0.940 & $<0.0001$ \\
\hline HERC $2 P 2$, & Hect domain and RLD 2 pseudogene 2 ; & & \\
\hline HERC $2 P 3$ & Hect domain and RLD 2 pseudogene 3 & 0.940 & $<0.0001$ \\
\hline$M E O X 2$ & Mesenchyme homeobox 2 & 0.933 & $<0.0001$ \\
\hline$S D C 2$ & Syndecan 2 & 0.929 & $<0.0001$ \\
\hline POSTN & Periostin, osteoblast specific factor & 0.921 & $<0.0001$ \\
\hline PLCB1 & Phospholipase C, $\beta 1$ (phosphoinositide-specific) & 0.920 & $<0.0001$ \\
\hline KCNJ8 & Potassium inwardly-rectifying channel, subfamily $\mathrm{J}$, member 8 & 0.918 & $<0.0001$ \\
\hline MAP4K4 & Mitogen-activated protein kinase kinase kinase kinase 4 & 0.917 & $<0.0001$ \\
\hline DOK5 & Docking protein 5 & 0.916 & $<0.0001$ \\
\hline STS & Steroid sulfatase (microsomal), isozyme $\mathrm{S}$ & 0.913 & $<0.0001$ \\
\hline HAS2 & Hyaluronan synthase 2 & 0.912 & $<0.0001$ \\
\hline$A P 1 G 1$ & Adaptor-related protein complex $1, \gamma 1$ subunit & 0.911 & $<0.0001$ \\
\hline
\end{tabular}

B, Negative differentially expressed genes

\begin{tabular}{|c|c|c|c|}
\hline Symbol & Gene title & Correlation & P-value \\
\hline$T F D P 1$ & Transcription factor Dp-1 & -0.894 & $<0.0001$ \\
\hline OSBPL9 & Oxysterol binding protein-like 9 & -0.806 & $<0.0001$ \\
\hline PLAA & Phospholipase A2-activating protein & -0.796 & $<0.0001$ \\
\hline BTF3 & Basic transcription factor 3 & -0.795 & $<0.0001$ \\
\hline$P P W D 1$ & Peptidylprolyl isomerase domain and WD repeat containing 1 & -0.792 & $<0.0001$ \\
\hline$L A R S$ & Leucyl-tRNA synthetase & -0.792 & $<0.0001$ \\
\hline NDUFA8 & $\begin{array}{l}\text { NADH dehydrogenase (ubiquinone) } 1 \alpha \text { subcomplex, } 8 \text {, } \\
19 \mathrm{kDa}\end{array}$ & -0.790 & $<0.0001$ \\
\hline ERAP2 & Endoplasmic reticulum aminopeptidase 2 & -0.787 & $<0.0001$ \\
\hline PTPN4 & $\begin{array}{l}\text { Protein tyrosine phosphatase, non-receptor type } 4 \\
\text { (megakaryocyte) }\end{array}$ & -0.784 & $<0.0001$ \\
\hline$H M G C R$ & 3-hydroxy-3-methylglutaryl-CoA reductase & -0.781 & $<0.0001$ \\
\hline TAF9 & TAF9 RNA polymerase II, TBP-associated factor, $32 \mathrm{kDa}$ & -0.777 & $<0.0001$ \\
\hline KLF5 & Kruppel-like factor 5 (intestinal) & -0.774 & $<0.0001$ \\
\hline$X R C C 4$ & $\begin{array}{l}\text { X-ray repair complementing defective repair in Chinese } \\
\text { hamster cells } 4\end{array}$ & -0.769 & $<0.0001$ \\
\hline MSH3 & MutS homolog 3 & -0.762 & $<0.0001$ \\
\hline$F B L$ & Fibrillarin & -0.753 & $<0.0001$ \\
\hline
\end{tabular}

tRNA, transfer RNA; NADH, nicotinamide adenine dinucleotide (reduced form); CoA, coenzyme A; TBP, TATA box-binding protein.

\section{Results}

Antitumor efficacy of ribavirin. Seven malignant glioma cell lines were treated with $0.1-1,000 \mu \mathrm{M}$ ribavirin and cultured for $72 \mathrm{~h}$. The proliferated cells were trypsinized and counted using a Z1 Coulter Counter ${ }^{\circledR}$. As demonstrated by Fig. 1, the growth of all of the cell lines was inhibited by ribavirin in a dose-dependent manner, although the sensitivity of each cell line to ribavirin varied. The $\mathrm{IC}_{50}$ of ribavirin for five of the malignant glioma cell lines (A-172, AM-38, T98G, U-87MG and $\mathrm{YH}-13$ ) was $<100 \mu \mathrm{M}$, whereas, the $\mathrm{IC}_{50}$ for the other two cell lines (U-138MG and U-251MG) was $>250 \mu \mathrm{M}$ (Table I).

Identification of discriminatory genes for ribavirin sensitivity. Of the 22,277 probe sets, genes that were not expressed were omitted, therefore, 16,913 probe sets were available for 
subsequent analysis. The expression profiling data for the malignant glioma cell lines were consistent with data from previous reports $(10,11)$. Various genes that were expressed in the seven malignant glioma cell lines included in the present study were observed to be upregulated or downregulated relative to ribavirin sensitivity. The negative and positive correlation values of the association between gene expression level and ribavirin sensitivity are indicated in Table II by the Pearson's correlation coefficient and the corresponding P-value. The gene expression levels of PDGFRA and TFDPI indicate the greatest positive and negative correlation with ribavirin sensitivity, respectively (Fig. 2).

Cell cycle distribution analysis. To clarify the antitumor efficacy of ribavirin on malignant glioma cells, alterations in the cell cycle distribution were examined using U-87MG cells treated with ribavirin. Unsynchronized cells were treated with $10 \mu \mathrm{M}$ ribavirin for 4,8 and $24 \mathrm{~h}$. The harvested cells were fixed using ethanol, treated with RNase A and stained with PI for analysis by fluorescence-activated cell sorting (FACS; Fig. 3A). Histograms presenting the FACS data (Fig. 3B) demonstrate an increase in the population of cells in the G0/G1 phase following treatment with ribavirin, with time-lapse indicating that the antitumor efficacy of ribavirin results from the accumulation of cells in the G0/G1 phase.

\section{Discussion}

To the best our knowledge, the current study is the first to demonstrate that ribavirin inhibits the growth of malignant glioma cells. Ribavirin was initially developed as an antiviral agent against RNA and DNA viruses and is predominantly used, in combination with IFN, as a treatment strategy for HCV infection (2). Although ribavirin was expected to be developed as a therapeutic treatment for various other viral infections, its clinical use has thus far been restricted to the treatment of $\mathrm{HCV}$ infections. Interest in the antitumor efficacy of ribavirin has been increasing due to its ability to inhibit IMPDH and eIF4E $(1,6)$. Furthermore, the antitumor efficacy of ribavirin has been reported in the treatment of breast cancer and leukemia $(5,9)$.

The antitumor efficacy of ribavirin on malignant glioma cell lines was measured and it was revealed that the growth of these cell lines was inhibited by ribavirin in a dose-dependent manner. Of the seven malignant glioma cell lines investigated in the current study, the $\mathrm{IC}_{50}$ of five of these cell lines (A-172, AM-38, T98G, U-87MG and YH-13) was $<100 \mu \mathrm{M}$ and the $\mathrm{IC}_{50}$ of the other two glioma cell lines (U-138MG and U-251MG) was $>250 \mu \mathrm{M}$. Based on these data, the malignant glioma cell lines can be divided into ribavirin-effective and ribavirin-resistant groups. The genes that were positively and negatively correlated with ribavirin sensitivity are listed in Table II. To the best of our knowledge, none of the genes identified in the current study were previously expected to be associated with ribavirin or chemotherapy sensitivity. TFDPI has been associated with the cell cycle and seven genes (PDGFRA, ZEP36L2, STS, TFDP1, HAS2, FBL and KLF5) have previously been associated with cell proliferation. In the present study, PDGFRA demonstrated the greatest positive correlation between gene expression level and the $\mathrm{IC}_{50}$ of ribavirin. Platelet-derived growth factor (PDGF) is a major mitogen for glial cells and connective tissue, and is key in the development of the central nervous system, wound healing, inflammation and neoplasia (12). PDGF receptor $\alpha$ is the cell surface receptor of PDGF. PDGFRA mRNA overexpression was detected in the low- and high-grade astrocytomas (13) and PDGFRA amplification is typical in the signaling pathway that results in the development of secondary glioblastoma (14). PDGFRA amplification is important for subgroup classification of malignant gliomas. To the best our knowledge, PDGFRA amplification and overexpression has not been reported, with regard to its association with chemotherapy sensitivity. However, although the association between rebavirin and PDGFRA amplification is unclear, we hypothesize that PDGFRA expression may be involved in the antitumor efficacy of ribavirin. Although it has been reported that ribavirin has an effect on the types of breast cancer that overexpress eIF4E, eIF4E was not extracted and analyzed in the present study as eIF4E is upregulated in all high-grade astrocytic tumors (15) and hence all malignant glioma cell lines. Furthermore, IMPDH was not included in the present study as it was reported that ribavirin inhibits leukemic cell proliferation through multiple signaling pathways (16), therefore, ribavirin may act on malignant glioma cells via mechanisms that do not involve eIF4E or IMPDH.

Finally, a cell cycle analysis of the harvested U-87MG cells, which were treated with ribavirin revealed that cells accumulated at the G0/G1 boundary; however, the sub-G1 population, which indicates apoptotic cells, did not increase. Vallée et al (17) reported that, in a melanoma cell line, the cell cycle was blocked in the G0/G1 phase by treatment with $100 \mu \mathrm{M}$ ribavirin. The results of the present study also indicate that ribavirin is important in the inhibition of cell growth in malignant glioma by inducing G0/G1 arrest. To the best of our knowledge, no studies have reported that ribavirin alone is able to induce apoptosis in malignant glioma cells. However, Schlosser et al (18) reported that the number of human hepatoma apoptotic cells was increased by treatment with $50 \mu \mathrm{M}$ ribavirin plus $\alpha$-IFN. This synergistic effect is also expected to occur in malignant glioma cells treated with ribavirin combined with IFN or temozolomide (TMZ).

The present standard postoperative treatment strategy for malignant glioma is TMZ plus radiation and the median treatment survival time is 14.6 months (19). The results of the current study indicate that ribavirin may present as a novel agent for malignant glioma chemotherapy and that PDGFRA expression levels may be a significant marker of the antitumor efficacy of ribavirin against malignant gliomas.

\section{References}

1. Sidwell RW, Huffman JH, Khare GP, Allen LB, Witkowski JT and Robins RK: Broad-spectrum antiviral activity of Virazole: 1-beta-D-ribofuranosyl-1,2,4-triazole-3-carboxamide. Science 177: 705-706, 1972

2. Fried MW, Shiffman ML, Reddy KR, et al: Peginterferon alfa-2a plus ribavirin for chronic hepatitis $\mathrm{C}$ virus infection. N Engl J Med 347: 975-982, 2002.

3. Yamada Y, Natsumeda Y and Weber G: Action of the active metabolites of tiazofurin and ribavirin on purified IMP dehydrogenase. Biochemistry 27: 2193-2196, 1988.

4. Jackson RC, Weber G and Morris HP: IMP dehydrogenase, an enzyme linked with proliferation and malignancy. Nature 256: 331-333, 1975. 
5. Assouline S, Culjkovic B, Cocolakis E, et al: Molecular targeting of the oncogene eIF4E in acute myeloid leukemia (AML): a proof-of-principle clinical trial with ribavirin. Blood 114: 257-260, 2009.

6. Kentsis A, Topisirovic I, Culjkovic B, Shao L and Borden KL: Ribavirin suppresses eIF4E-mediated oncogenic transformation by physical mimicry of the 7-methyl guanosine mRNA cap. Proc Natl Acad Sci USA 101: 18105-18110, 2004.

7. Culjkovic B, Topisirovic I and Borden KL: Controlling gene expression through RNA regulons: the role of the eukaryotic translation initiation factor eIF4E. Cell Cycle 6: 65-69, 2007.

8. Borden KL and Culjkovic-Kraljacic B: Ribavirin as an anti-cancer therapy: acute myeloid leukemia and beyond? Leuk Lymphoma 51: 1805-1815, 2010.

9. Pettersson F, Yau C, Dobocan MC, et al: Ribavirin treatment effects on breast cancers overexpressing eIF4E, a biomarker with prognostic specificity for luminal B-type breast cancer. Clin Cancer Res 17: 2874-2884, 2011.

10. Yoshino A, Ogino A, Yachi K, et al: Gene expression profiling predicts response to temozolomide in malignant gliomas. Int J Oncol 36: 1367-1377, 2010.

11. Yoshino A, Tashiro S, Ogino A, et al: Gene expression profiles predicting the response to IFN- $\beta$ and a combination of temozolomide and IFN- $\beta$ in malignant gliomas. Int J Oncol 39: 529-542, 2011.

12. Heldin $\mathrm{CH}$ and Westermark B: Platelet-derived growth factor: mechanism of action and possible in vivo function. Cell Regul 1 : $555-566,1990$
13. Hermanson M, Funa K, Koopmann J, et al: Association of loss of heterozygosity on chromosome $17 \mathrm{p}$ with high platelet-derived growth factor alpha receptor expression in human malignant gliomas. Cancer Res 56: 164-171, 1996.

14. Kleihues P, Burger PC and Aldape KD. Glioblastoma. In: WHO Classification of Tumours of the Central Nervous System. Louis DN, Ohgaki H, Wiestler OD and Cavenee WK (eds). 4th edition. IARC Press, Lyon, France, pp33-49, 2007.

15. Gu X, Jones L, Lowery-Norberg M and Fowler M: Expression of eukaryotic initiation factor 4E in astrocytic tumors. Appl Immunohistochem Mol Morphol 13: 178-183, 2005.

16. Kökény S, Papp J, Weber G, Vaszkó T, Carmona-Saez P and Oláh E: Ribavirin acts via multiple pathways in inhibition of leukemic cell proliferation. Anticancer Res 29: 1971-1980, 2009.

17. Vallée S, Fouchier F, Braguer D, Marvaldi J and Champion S: Ribavirin-induced resistance to heat shock, inhibition of the Ras-Raf-1 pathway and arrest in G(1). Eur J Pharmacol 404: 49-62, 2000.

18. Schlosser SF, Schuler M, Berg CP, et al: Ribavirin and alpha interferon enhance death receptor-mediated apoptosis and caspase activation in human hepatoma cells. Antimicrob Agents Chemother 47: 1912-1921, 2003.

19. Stupp R, Mason WP, van den Bent MJ, et al; European Organisation for Research and Treatment of Cancer Brain Tumor and Radiotherapy Groups; National Cancer Institute of Canada Clinical Trials Group. Radiotherapy plus concomitant and adjuvant temozolomide for glioblastoma. N Engl J Med 352: 987-996, 2005. 